\title{
Nanomodified compositions based on finely dispersed binders for soil reinforcement
}

\author{
Lev Alimov ${ }^{1}$,Igor Kharchenko, ${ }^{2,}$ and Viktor Voronin ${ }^{1}$ \\ ${ }^{1}$ Moscow State University of Civil Engineering, Yaroslavskoeshosse, 26, Moscow, 129337, Russia
}

\begin{abstract}
Theoretical prerequisites on the possibility of improvement of physical and mechanical properties of soils at underground space development, their stability at different aggressive actions by means of their structure impregnation with nanomodified suspensions on the basis of especially finely dispersed mineral binders are developed. The features of influence of plasticizers on penetration ability and sedimentation stability of suspensions are revealed. Soil body reinforcement after its impregnation may achieve considerable values, which is related to the features of interaction of components of impregnating composition with extended surface of soil pore space.
\end{abstract}

\section{Introduction}

Reinforcement of soil-concrete bodies and improvement of reliability of cutoff curtains at underground space development of urban areas, their stability at different aggressive actions is achieved by means of implementation of impregnating compositions on the basis of especially finely dispersed mineral binders (EFDB). Impregnating compositions intended for soil modification represents the disperse systems, where binder solid particles are suspended in liquid disperse medium [1]. Particle size range varies from tenths of $\mathrm{mm}$ to particles less than $10^{-7} \mathrm{~m}$. EFDB particle size does not exceed $16 \mu \mathrm{m}$. At interaction with water these particles almost completely hydrate to form the colloidal solutions with particle size of 1-100 nm. Herewith, some particles form crystal hydrate compounds when dissolved [2].

In order to achieve high density and homogeneity of soil-concrete prepared by impregnation of its structure, it is necessary to achieve high penetration ability of injection mortar prepared on EFDB basis. According to conducted research despite of high level of water content in suspension having $\mathrm{W} / \mathrm{B}=2,0-5,0$, plasticizer content greatly affects both suspension penetration ability and its sedimentation stability (Fig.1).

\footnotetext{
*Corresponding author: iharcenko@mail.ru
} 


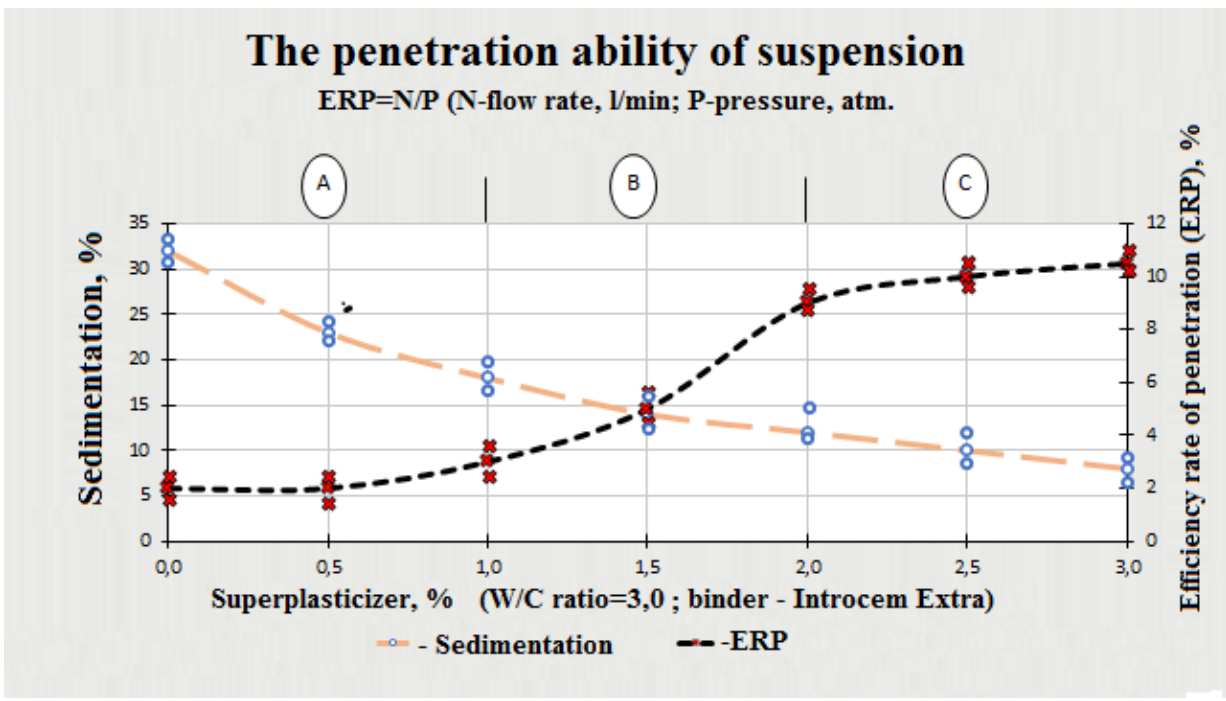

Fig.1. Suspension penetration ability

However, high viscosity does not guarantee suspension high penetration ability. Sedimentation stability affects the penetration efficiency factor. Herewith, penetration ability of injection mortar was evaluated by its penetration efficiency factor (or ERP):

$\mathrm{ERP}=\mathrm{N} / \mathrm{P}$, where

$\mathrm{P}$ - the injection pressure, bar;

$\mathrm{N}$-the injection rate, $1 / \mathrm{min}$.

\section{Materials, methods and mixture proportioning}

During work the following materials were used:

1. Finely dispersed mineral binder(EFDB) - "Microdur - R" with high content of blastfurnace slag with Portland cement clinker. Content of particles less than $5 \mu \mathrm{m}$ is more than $95 \%$. Maximum grain size does not exceed $16 \mu \mathrm{m}$.

2. SuperplasticizerC-3 - the product of condensation of naphthalene sulphonic acid and formaldehyde, light-brown powder with w/w of active substance of $70 \%$, of ash of $37 \%$, and of formaldehyde of $0,1 \%$.

Research methods of EFDB include an assessment of the penetration ability and sedimentation depending on water-cement ratio and rate super plasticizer. Physico-chemical testing of cement stone and soil-concrete included $\mathrm{x}$-ray fluorescence analysis and differential thermal analysis and electron microscopy.

According to conducted research, the efficiency of influence of such plasticizer as, e.g., "C3 ", is revealed at its consumption of $1 \%-2 \%$ of EFDB weight. Naturally, for other types of plasticizers and super plasticizers it is necessary to conduct a separate research [3]. However, despite of distinction in properties of plasticizers and super plasticizers by different manufacturers, the mechanism of their influence can be considered in the scheme shown in (Fig.2). 


\section{Dispersion of the binder in the aquatic environment}

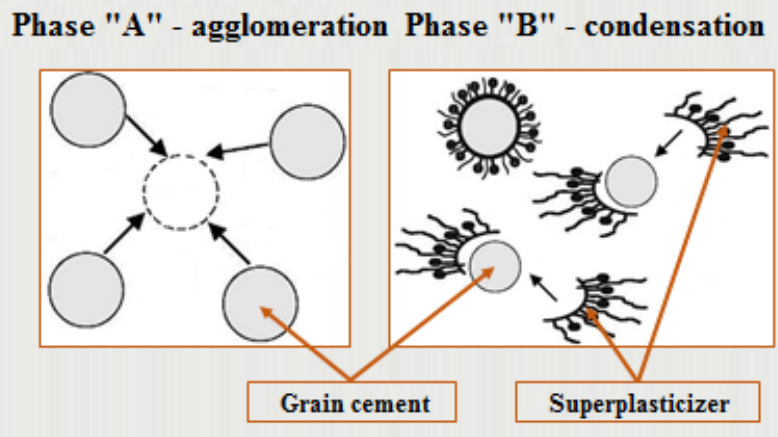

Phase "C"- dispersion

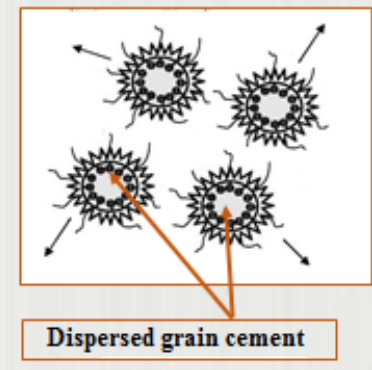

Fig.2. EFDB dispersion scheme at introduction of plasticizers.

The scheme of EFDB grains interaction with plasticizer in water medium may be conventionally divided into three phases: phase "A" - agglomeration; phase " $B$ " - condensation; phase "C" - dispersion. At introduction of mineral powder into mixing water composition due to substantially extended specific surface separate EFDB grains tend to agglomerative coarsening $[4,5]$.

In order to prevent EFDB grains agglomeration at mixing with water, high-speed mixers are applied with shaft speed of not less than 1500 - $2000 \mathrm{rpm}$. Herewith, mixer tank is initially filled with design amount of mixing water, and after that during continuous rotation of mixer the EFDB powder is supplied evenly. With the purpose of more qualitative EFDB grains distribution in mixing water volume the mixer is equipped with a special toothed multi-blade hopper, and the mixer walls are equipped with special deflecting blades. Herewith, at optimum correlation between the volume of prepared mix and electric motor power, the duration of suspension preparation is $90-120 \mathrm{sec}$.

Thus, the soil is modified with the suspensions with high water content at water-solid ratio of more than four. Herewith the strength of modified soil may reach considerable values (up to $25 \mathrm{MPa}$ ), which is first of all related to the features of interaction of impregnating composition components with extended area of soil pore space.

\section{Results and Discussions}

Colloidal solution in capillary-porous body in confined space is restricted with the external surface of pore space. Substance properties in this phase contact area with the thickness of several atom or molecule diameters being directly on the surface, differ from the properties inside the phase volume. Inside the pure substance volume in solid, liquid or gaseous state any molecule is surrounded with similar molecules. Within the boundary layer the molecules interact either with different number of molecules, or with molecules of different chemical nature. The greater is the difference in intensity of intermolecular forces acting in every phase, the higher is the potential energy in phase contact area. The efficiency of introduction of nanosystem with particles less than $10^{-7}$ mto porous space of material possessing huge expanded surface is generally based on the reduction of energy threshold of early formation of crystalline hydrates from water solution saturated with ions of cement clinker minerals as the result of its interaction with mixing water. I.e., in this case the high level of surface energy of nanoparticles is used, which may act as crystallization centers boosting the process of crystalline hydrates formation and providing strengthening of capillary-porous material. Hydrate nuclei appear in 
the vicinity of pore space surface, because their formation within the solution volume (in liquid medium) is a less energetically advantageous process $[6,7]$.

Here occur the colloid-chemical processes, as the result of which are formed the colloidal gels. Particles of the new phases form highly swollen colloids due to creation of solvation shells around them. As the result, they increase in volume (swell out) and after a while they come in close contact with each other, forming coagulative nodes. From oversaturated liquid of cementwater suspension the three-dimensional crystalline nuclei are evolved with the diameter of $10^{-}$ ${ }^{9} \mathrm{~m}$, which then rapidly coarsen (coagulate) up to the size of about $10^{-7} \mathrm{~m}$. They mainly precipitate on the surface of the pore space $[8,9]$.

Particles of newgrowthsare characterized with high dispersion ability. Their specific surface amounts $200-400 \mathrm{~m}^{2} / \mathrm{g}$, i.e. the crystals of hydrated calcium silicates reach the thickness only of 2-3 elementary layers. At specified crystal thickness, half to $2 / 3$ of all their atoms and ions are on the surface and, consequently, they are characterized with the presence of free energy stipulating the bonds between the particles of newgrowths and solidification of the entire system.

Hydrated calcium silicates possess the ability to bind a considerable amount of water, and when the growing crystalline hydrates get closer to a distance of several diameters of water molecules, they coalesce with each other with their crystalline nuclei of the same phases as the result of fluctuation consolidating of molecules of dissolved substances [10].

In pore space a crystalline aggregate is formed consisting of crystals of hydrated calcium aluminate, Portlandite, hydrated sulfoaluminate and hydrated calcium sulfoferrite, as well as of gel structure, in which the submicrocrystals of hydrated calcium silicate are a dispersing phase, which is confirmed with the research of stone structure by means of electron microscopy.

At impregnating injection of sandy soils a contact area occurs as the result of physical and chemical processes occurring between the modified suspension and pore surface. Contact layer differs markedly in its composition and structure from the cement stone incracks and cavities. The research of contact layer by means of electron scanning microscope revealed the presence at the surface between the pore space and forming composite of Portlandite, ettringite and aggregates of C-S-H-gel.

The composition of contact layer depends on physical and chemical activity of soil components. The research of reaction occurring on the surface of different soils: acid soils with content of $\mathrm{SiO}_{2}=65 \%$ (granite, granulite, pyroxene-quartz porphyry); neutral soils with content of $\mathrm{SiO}_{2}=52-65 \%$ (augite-porphyry) and basic soils with content of $\mathrm{SiO}_{2}=52 \%$ (diabase, basalt, disintegrated basalt), as well as quartz, albite, orthoclase and carbonate rocks (dolomite, dolomitic limestone) revealed that at early stages basic rocks bind slightly more limestone than the acid ones.

The analysis of structure of solidified stone was conducted by means of X-ray phase analysis and differential thermal analysis methods. The degree of hydration of finely dispersed binders was determined on the basis of the degree of hydration of $\mathrm{C}_{3} \mathrm{~S}$. X-ray phase analysis showed that the degree of hydration amounted about $85 \%$. The amount of hydrated lime can be inferred by peak intensity $\mathrm{d}=4,902 \mathrm{~A}$. In cement stone is further observed the formation of lime carbonate of line $(\mathrm{d}=3,03,2,492,2,283,2,294,1,904,1,874,1,628,1,604,1,525,1,485 \mathrm{~A})$ (Fig.3). 


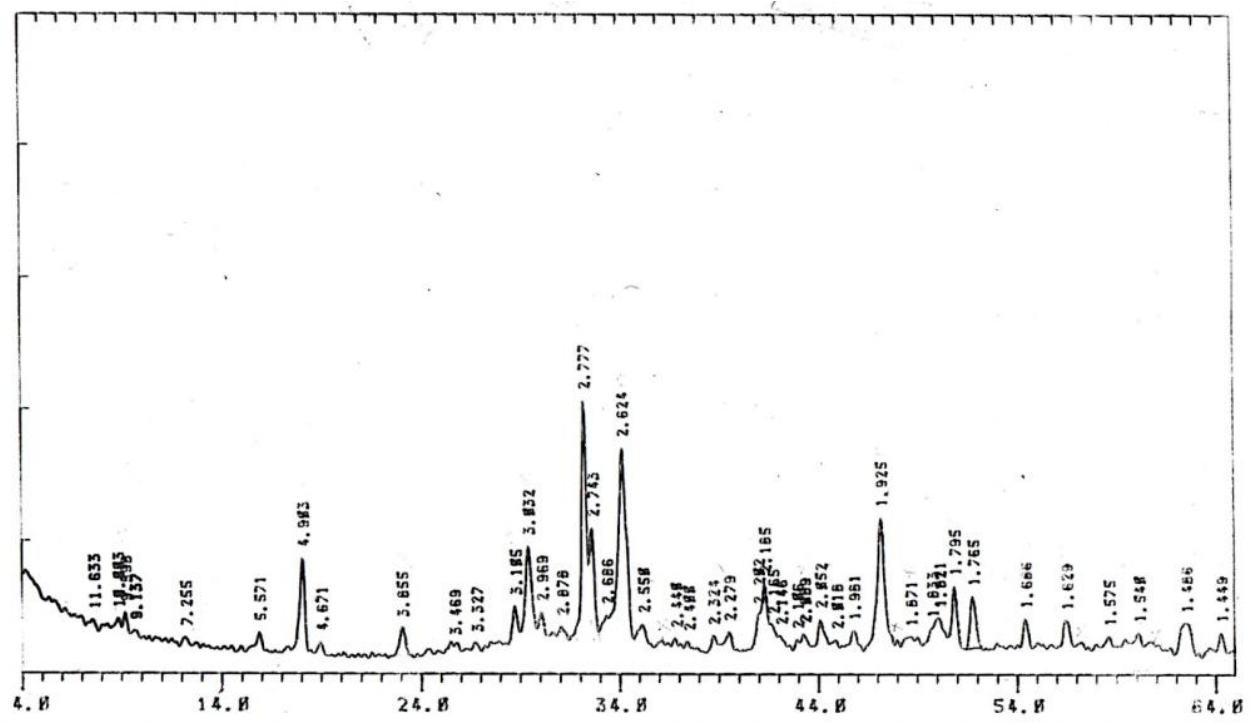

Fig.3. X-ray pattern of a sample of cement stone on EFDB basis.

In termograms of samples the endothermic effects are observed at the temperature of $100-$ $120{ }^{\circ} \mathrm{C}$, which point to the removal of loosely bound adsorption water and partially of hydration water from ettringite (Fig. 4). Dehydration processes within this temperature range are accompanied with the greatest weight loss, which is fixed in TG curve. Endoeffect within the temperature range of $840-860^{\circ} \mathrm{C}$ marked in both thremogravigrams reflects the calcite dehydration. Exothermic effect at the temperature of $910-920{ }^{\circ} \mathrm{C}$ is usually referred to tobermorite hydration. Apparently, differential thermal analysis curves confirm the results of Xray phase analysis.

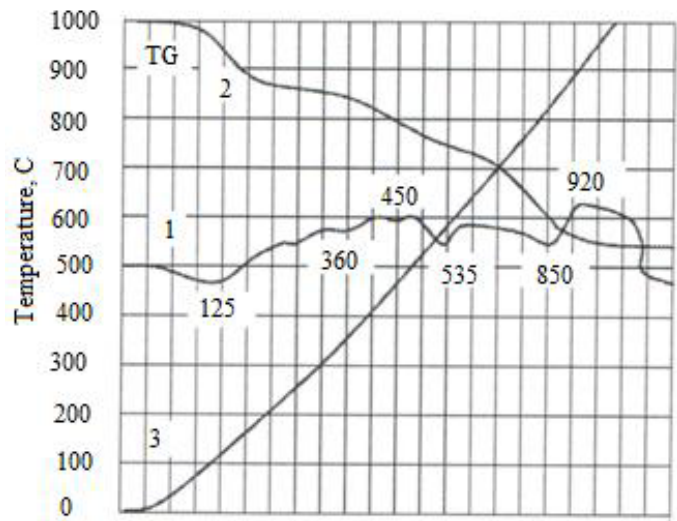

Fig. 4. Thermogram of samples of cement stone on EFDB basis

Particular interest is in the comparative analysis of pore structure of soil-concrete body formed by means of structure impregnating with injection mortar and the mechanical mixing of soil and suspension, which demonstrated significant difference bothin value and in volume and nature of capillary-porous structure. (Fig. 5). 

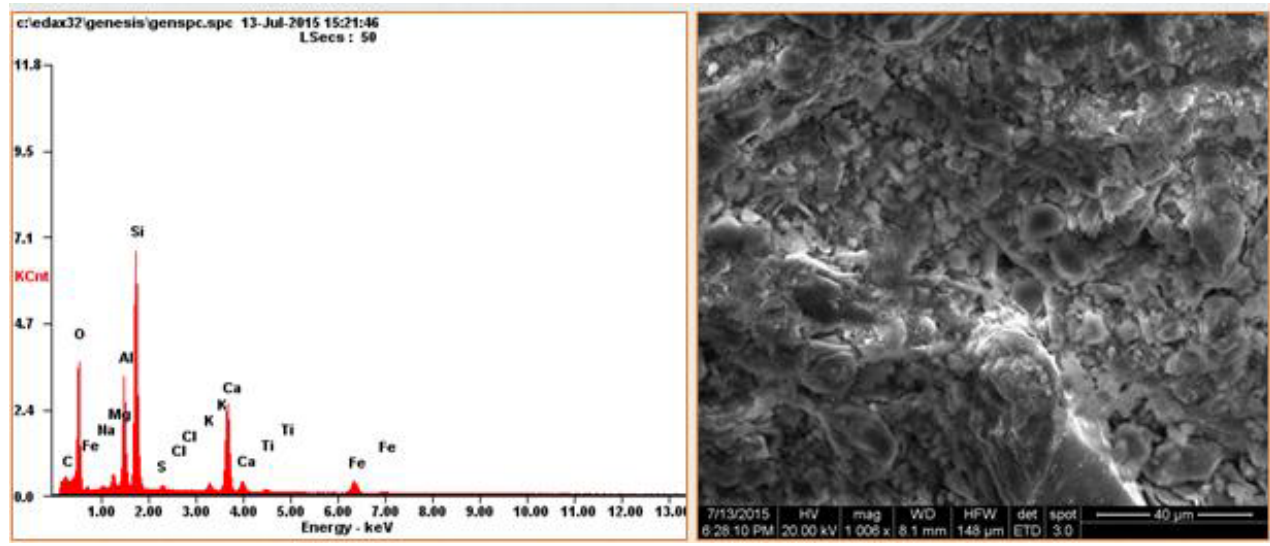

Fig.5. Soil-concrete microstructure.

\section{Conclusion}

The total pore volume of soil-concrete after impregnation is more than 2,7 times less than of soil-concrete prepared by mechanical mixing of soil and suspension. Herewith, if soil-concrete prepared by mechanical mixing of soil and suspension has mainly open pores and capillaries (more than $85 \%$ ), and the portion of closed pores is about $15 \%$ of total pore volume, for the structure of soil-concrete prepared by impregnating it is vice versa, i.e. pores and capillaries have mainly closed nature. Formed dense and impenetrable structure of soil-concrete prepared by impregnating of soil structure has average density comparable with average density of fine grained concrete, while average density of soil-concrete prepared by mechanical mixing corresponds to average density of aerated fine grained concrete.

Solidified injection mortar within the volume of capillaries and pores of soil-concrete body is represented with a dense structure containing beside the hydration products the grains of microfiller forming the structures of almost flawless microconcrete. Contact area between the solidified injection mortar and soil grains also has qualitative almost flawless structure providing reliable joint work of all the elements of soil-concrete structure.

Thus, the theoretical prerequisites are developed, allowing formulation of possibility of considerable improvement in physical and mechanical characteristics of soils by means of their structure impregnating with nanomodified suspensions on the basis of finely dispersed binders with maximum grain size of $5-16 \mu \mathrm{m}$ forming after hydration the colloidal solutions with particle size of 1-100 nm, possessing high surface energy level and acting as crystallization centers both on the surface of pore space of injected material and within the volume of capillary-porous structure, facilitating the formation of crystalline aggregate consisting of crystals of hydrated calcium aluminate, Portlandite, hydrated sulfoaluminate and hydrated calcium sulfoferrite, as well as of gel structure, in which submicrocrystals of hydrated calcium silicates act as dispersing phase.

\section{References}

1. I. Mehdipour, K. H. Khayat, Cem. and Conc. Comp.,78, 120(2017)

2. I. J. Harcschenko, D. Teichert, V. Ruder, Patent EP 0696558B1 Superfine cement binder mixture, process for its preparation (1998)

3. I. J. Harcschenko, D. Teichert, V. Ruder, Patent DE 4447575C1 (1998)

4. A. I. Harcenko, D. A.Bagenov, Z. A. Sugkoev, 19 Internationale Baustoftagung 
"IBAUSIL", (Weimar, 2015)

5. I.Ya. Kharchenko, S.A.Kryvchun, A. I Kharchenko. $1^{\text {st }}$ International research and practice conference INTERMETRO "Prospects for further development o subway within the conditions of intensive implementation of technical innovations» (Moscow, 2015)

6. I. Ya. Kharchenko, V. EMerkin, Transport construction (in Russian - Transportnoe stroitelstvo), 1, 6 (2015)

7. O. Larsen, Modern science and innovation (in Russian Sovremennayanaukaiinnovatsii), 2(14), 140 (2014)

8. I. Ya Kharchenko et al. Sub-bases, foundations and soil mechanics (in Russian osnovaniya, fundamenty i mekhanika gruntov), 1, 2(2015)

9. M. I. Bazhenov, A. I. Kharchenko, I. Ya Kharchenko, Building materials (in Russian Sroitelnyestmaterialy), 10, 65(2012)

10. Yu. M. Bazhenov, L. A. Alimov, V. V. Voronin, Structure and properties of concretes with nanomodifiers on the basis of technogenic wastes (Moscow, 2013) 